Revue internationale P.M.E.

Économie et gestion de la petite et moyenne entreprise

\title{
Une nouvelle vedette : la PME de services en région périphérique
}

\section{Annette Ribordy, Eugène Kaciak et Azim Kassaramali Bhimani}

Volume 3, numéro 3-4, 1990

URI : https://id.erudit.org/iderudit/1007985ar

DOI : https://doi.org/10.7202/1007985ar

Aller au sommaire du numéro

Éditeur(s)

Presses de l’Université du Québec

ISSN

0776-5436 (imprimé)

1918-9699 (numérique)

Découvrir la revue

Citer cet article

Ribordy, A., Kaciak, E. \& Kassaramali Bhimani, A. (1990). Une nouvelle vedette : la PME de services en région périphérique. Revue internationale P.M.E., 3(3-4), 345-366. https://doi.org/10.7202/1007985ar
Résumé de l'article

Cet article présente les résultats d'une enquête conduite auprès de la communauté francophone des affaires du nord-est de l'Ontario, au sujet des perceptions reliées à l'évolution économique de la région. Les données recueillies ont été traitées par l'analyse de correspondance multiple, une technique multivariée qui permet de présenter graphiquement les relations existant entre plus de deux variables qualitatives. Cette analyse a permis de dégager trois profils d'attitudes : à un extrême, les très petites compagnies du secteur manufacturier et les membres de la fonction publique manifestent des attitudes pessimistes. A l'opposé, les membres du secteur des services, particulièrement les propriétaires et les professionnels à l'emploi de petites et très jeunes compagnies de services et de franchises se montrent extrêmement satisfaits de l'évolution économique de la région. Entre les deux, les modérés ou indécis se retrouvent parmi les superviseurs de très grandes entreprises et parmi les gérants de coopératives.

Ces résultats viennent confirmer les tendances retrouvées dans l'économie générale où l'on assiste, depuis les années 1970, à un recul sensible du secteur industriel et à une explosion des services comme générateurs d'emploi. Enfin, l'étude a révélé l'émergence d'une nouvelle classe de gens d'affaires, celle des directeurs et propriétaires de franchises et de PME des services qui, durant une période de changements économiques, on su percevoir les opportunités et en tirer parti.
Ce document est protégé par la loi sur le droit d'auteur. L'utilisation des services d’Érudit (y compris la reproduction) est assujettie à sa politique d'utilisation que vous pouvez consulter en ligne.

https://apropos.erudit.org/fr/usagers/politique-dutilisation/ 


\title{
Une nouvelle vedette : la PME de services en région périphérique
}

\author{
Annette RIBORDY* \\ Université Laurentienne \\ Eugène KACIAK \\ Université Brock \\ Azim KASSARAMALI BHIMANI* \\ Université Laurentienne
}

\begin{abstract}
RÉSUMÉ
Cet article présente les résultats d'une enquête conduite auprès de la communauté francophone des affaires du nord-est de l'Ontario, au sujet des perceptions reliées à l'évolution économique de la région. Les données recueillies ont été traitées par l'analyse de correspondance multiple, une technique multivariée qui permet de présenter graphiquement les relations existant entre plus de deux variables qualitatives. Cette analyse a permis de dégager trois profils d'attitudes : àun extrême, les très petites compagnies du secteur manufacturier et les membres de la fonction publique manifestent des attitudes pessimistes. A l'opposé, les membres du secteur des services, particulièrement les propriétaires et les professionnels à l'emploi de petites et très
\end{abstract}

* Annette Ribordy enseigne le marketing à l'Université Laurentienne de Sudbury (Ontario). Elle possède un M.B.A. de l'Université Laurentienne et poursuit ses études de doctorat à l'École des H.E.C. de l'Université de Lausanne. Ses recherches et publications portent sur divers aspects de l'économie nord-ontarienne.

Eugène Kaciak est professeur de statistique et méthodes quantitatives à l'Université Brock de St. Catharines (Ontario). Il détient un doctorat de l'Université de Varsovie. II est l'auteur de plusieurs publications sur divers aspects des statistiques appliquées, de la programmation linéaire et des probabilités subjectives.

Azim Kassaramali Bhimani est professeur de finance. Il a obtenu une maîtrise (M.B.A.) à l'Université de Western Ontario. Ses intérêts de recherche portent sur l'aspect financier dans les industries à technologie avancée.

Adresse : École de l'administration et du commerce, Division de recherche, Université Laurentienne, Chemin du lac Ramsey, Sudbury (Ontario), P3E 2 C6. 
jeunes compagnies de services et de franchises se montrent extrêmement satisfaits de l'évolution économique de la région. Entre les deux, les modérés ou indécis se retrouvent parmi les superviseurs de très grandes entreprises et parmi les gérants de coopératives.

Ces résultats viennent confirmer les tendances retrouvées dans l'économie générale où l'on assiste, depuis les années 1970, à un recul sensible du secteur industriel et à une explosion des services comme générateurs d'emploi. Enfin, l'étude a révélé l'émergence d'une nouvelle classe de gens d'affaires, celle des directeurs et propriétaires de franchises et de PME des services qui, durant une période de changements économiques, on su percevoir les opportunités et en tirer parti.

\section{ABSTRACT}

This article presents the results of a study conducted to examine North Eastern Ontario's francophone business community's perceptions of economic growth within the region. Multiple correspondence analysis, a multivariate technique that allows to demonstrate graphically relationships amongst several qualitative variables, was used to study the findings. Three sets of attitudes emerged out of this study : at one extreme, small manufacturing businesses and public sector employees portraide pessimistic attitudes. At the other extreme, service sector selfemployed and professionals in the very young, small, service sector businesses as well as franchise operators seemed highly optimistic about the economic scenario. The ain between" or «undecided" were the middle managers of large enterprises and the directors of cooperatives.

The results seem to confirm the general employment generating trends experienced by the general economy. Since the 1970"s, this trend has shown a decline of the industrial sector and an explosive growth of the service sector. Moreover, this study has highlighted the growth of a new abreed" of businesses - franchises and service sector small businesses-both of which have been able to capitalize on the opportunities that are present in this period of economic change.

\section{RESUMEN}

Este articulo presenta los resultados de una encuesta sobre las percepciones de la evolucion economica de la region nor-este de Ontario, desde el punto de vista del grupo de gentes de negocios de habla francesa.

Las respuestas fueron analyzadas por el metodo de correspondencias multiples, une tecnica multivariable que permite una representacion grafica de las relaciones entre mas de dos variables cualitativas.

El analisis permite agrupar las respuestas en tres neveles : por un lado, las pequeñas empresas de manufactura y los miembros de la funcion publica manifiestan actitudes pesimistas. Al otro lado, los miembros del sector de servicios, en particular los proprietarios y los profesionales que trabajan en companias de servicio nuevas y companias de franquicia se muestran sumamente satisfechos de la evolucion economica de la region. Entre ambos extremos, los supervisores y cuadros medios de las grandes empresas y los directores de organizaciones cooperativas forman el grupo moderado o indeciso.

Estos resultados confirman las tendencias de la economia regional que se vienen observando desde los años setenta : una baja importante en el sector industrial y una explosion del empleo en los grupos de servicios. Asimismo, este estudio señala la aparicion de directores y propretarios de negocios de servicios como un grupo nuevo que han sabido percibir y aprovechar las nuevas uportunidades de negocios. 


\section{Introduction}

Le glissement de l'économie vers une prédominance des secteurs tertiaire et quaternaire comme principaux générateurs de l'emploin'est pas un faitnouveau dans les économies de type occidental. Pourtant, la nature de cette transformation demeure confuse et les chercheurs ${ }^{1}$ ne s'entendent pas toujours sur les conséquences de ce changement sur la société et l'économie. D'une part, les services sont considérés comme un mal nécessaire, un travail improductif dont il faut comprimer le plus possible les coûts. D'un autre côté, ils sont vus comme créateurs de richesse, donc comme facteurs-clés de l'économie future (Barcet et Bonamy, 1985).

C'est ce changement de structure de l'emploi dans l'économie d'une région périphérique, traditionnellement vouée à l'alimentation des grandes régions industrielles en matières premières, que nous nous proposons d'étudier en examinant la perception qu'en ont les gens d'affaires de la région. Cette démarche devrait nous permettre, en plus de dégager le profil de la communauté des affaires de la région, d'ouvrir de nouvelles pistes en initiant des recherches sur d'autres régions de l'hinterland, stimulant, par le fait même, la réflexion sur l'effet des changements économiques.

\section{Les services en question}

Le secteur des services occupe une place de plus en plus grande dans la structure de l'emploi des pays industrialisés. A la fin de la dernière guerre, la force ouvrière canadienne était répartie à raison de $60 \%$ dans la production des biens et de $40 \%$ dans celle des services. Vers le milieu des années 1980 , les pourcentages étaient renversés avec $66 \%$ des emplois dans les services et $33 \%$ dans la production des biens (Chand, 1983, 1986). Cette tendance se poursuit aujourd'hui, puisque la proportion des emplois tertiaires atteint plus de $80 \%$ de la main-d'oeuvre active, à tel point que de Bandt (1985) se demande à quel moment le rapport de force ne va pas basculer complètement au profit des services.

Ce changement, amorcé pendant les années 1920, s'accélère après la fin de la deuxième guerre alors que les économies intérieures se développent (Noyelle, 1985) qu'on assiste à l'émergence de la consommation de masse, que le système de distribution s'organise à une grande échelle et que le profil économique prend une forme pyramidale. Au sommet, un nombre restreint de villes, que Christaller (1933) appelle les lieux centraux, contrôlent le pouvoir économique, l'industrie manufacturière et les sièges sociaux des grandes compagnies. Au niveau intermédiaire se situent les centres régionaux relativement peu industrialisés et axés principalement sur la distribution régionale; leur statut demeure mal défini parce qu'elles ne possèdent pas le réseau structuré des grandes villes (Cunha et Racine,

1 Le générique masculin employé ici n'a aucune volonté discriminatoire et ne vise qu’à l'allègement du texte. 
1984). Au bas de la pyramide, enfin, se retrouve un vaste territoire dans lequel les activités des petites villes sont orientées avant tout vers la satisfaction des besoins en tertiaire de leur région immédiate (Cunha et Racine, 1984). Cette dépendance des régions peu urbanisées n'est pas propre à l'Amérique du Nord, mais se retrouve aussi bien en Angleterre (Daniels, 1978), qu'en France (Philippe et Monnoyer, 1985), ou en Suisse (Bailly et Maillat, 1988), pour ne citer que quelques pays.

Dès les années 1970, l'économie commence à s'internationaliser et la concurrence des pays étrangers provoque un mouvement de modernisation et de rationalisation de l'industrie, d'où une diminution sensible des effectifs manufacturiers. Ce déclin est encore accentué par la récession qui affecte considérablement la consommation des biens durables tels les automobiles, l'ameublement, les produits électro-ménagers... (Chand, 1986).

Durant les années qui ont précédé la récession, le déficit des effectifs qui s'était manifesté dans le secteur manufacturier par rapport à celui des services, $s$ 'est encore accentué durant la crise, et la tendance s'est poursuivie par la suite, car tous les emplois créés dans l'économie canadienne depuis le début des années 1980 l'ont été dans le services (Picot, 1986; Chand, 1986). En provoquant le recul de l'industrie, la crise a donc mis en évidence les faiblesses des pays développés, démontrant à quel point les services sont mal compris et mal intégrés (Petit, 1985).

La répartition de l'emploi par secteurs d'activités remonte aux travaux de Clarke (1951) qui divisa l'emploi dans l'économie en trois catégories : le primaire, directement relié à l'exploitation des richesses naturelles et agricoles; le secondaire ou manufacturier; le tertiaire, qui regroupe toutes les activités ne pouvant être incluses dans les deux premières catégories. Depuis, un nombre important de chercheurs se sont penchés sur cette question complexe, adoptant ou remettant en question cette classification de l'emploi.

Un premier groupe, se fondant sur la classification de Clarke, voit ces changements structurels de l'emploi d'un oeil pessimiste. Cette croissance est considérée d'un point de vue de dé-industrialisation, de recul de l'emploi secondaire (Siniscalco, 1985).

La tertiarisation, cette économie des services basée sur les dépenses des ménageset sur la consommation, aurait des implications très sérieuses pour la société etl'économie. La première conséquence serait un recul des salaires moyens (Halami, 1989), puisqu'une qu'une très grande proportion de ces nouveaux emplois se retrouvent dans des activités où les revenus se situent au-dessous de la moyenne des salaires et dans les emplois à temps partiel dont le taux atteint au Canada 19,2\% contre $5.8 \%$ dans l'industrie (C.L.M.P.C., 1988). Ce sont des emplois qui relèvent du tertiaire banal, c'est-à-dire des services à la population ne requérant aucune qualification particulière, donc occupés par des catégories particulières de travailleurs : jeunes sans qualification, femmes retournant sur le marché du travail, immigrants. Par le fait même, ils tiennent peu de place dans le débat serviceproduction. Ils subissent ce que de Bandt (1985) appelle les «effets poubelle». Ils sont 
victimes des contrecoups des fluctuations de l'emploi dans l'industrie secondaire dont les «effectifs libérés sont refoulés vers les activités [de service] où les barrières à l'entrée sont faibles, voire nulles. Bien entendu, l'effet de rééquilibrage ainsi opéré dans les effectifs se répercute dans les niveaux de rémunération» (p. 219).

Face à ces catégories d'emplois au caractère marginal, sous-qualifiées et sous-payées, se retrouvent toutes les classes d'emplois dans les services, notamment celles des professionnels ou des gestionnaires qui sont rémunérées à des taux situés bien au-dessus de la moyenne. Il n'est donc pas étonnant que ce dualisme des services préoccupe les spécialistes (de Bandt, 1985; Noyelle, 1985; C.L.M.P.C., 1988).

Un autre sujet de préoccupation est souvent évoqué : celui de la productivité du secteur tertiaire. Selon le Centre canadien du marché du travail et de la productivité, entre 1950 et 1980, la croissance moyenne du rendement au Canada a été de 2,2 \% par année. Durant la même période, elle s'élevait à 4,3\% dans l'agriculture, à $3,1 \%$ dans l'industrie et à seulement $1 \%$ dans les services. Dans ce dernier secteur, le pourcentage s'est encore abaisséà 0,5\% par année entre 1981 et 1987 (C.L.M.P.C., 1988). Chang (1986) attribue la forte croissance du secteur des services à son taux moindre de productivité. Le problème majeur réside dans le fait que le secteur des services est essentiellement différent de celui des biens parce que le client participe à l'acte de production et qu'il est, par le fait même, difficile d'appliquer une théorie de la valeur au domaine des services (de Bandt, 1985). Mais à long terme, le fait de conserver et de protéger les emplois à faible salaire compromet les capacités de création de richesse d'un pays (Conseil du Premier Ministre, 1988).

Enfin, le rôle des services dans les échanges extérieurs retient l'attention des auteurs. Dans la plupart des pays, les services tendent à être considérés comme difficilement exportables parce que hautement réglementés et protégés en raison d'exigences politiques et stratégiques (Rabier et Sipek, 1985; Petit, 1985). C'est là une question qui préoccupe particulièrement les membres du Conseil du Premier Ministre ontarien (1988) chargés d'analyser la compétitivité industrielle de la province face à la globalisation des marchés. A l'exception de quelques industries internationales en consultation et en ingénierie, les services demeureraient essentiellement à caractère domestique et contiendraient peu de potentiel de croissance qui ne soit pas une demande dérivée de la production des biens. C'est pourquoi on considère que les industries primaires et manufacturières conservent leur importance pour la santé générale de l'économie, car elles sont responsables des exportations qui créent la majorité des revenus utilisés par les consommateurs pour acheter les services. En fait, cette préoccupation est motivée principalement par notre balance des paiements continuellement déficitaire.

Un second courant de pensée émet des opinions qui vont à l'encontre des premières et propose une redéfinition des services. Ces demiers, en permettant d'augmenter l'efficacité de l'appareil productif, d'accroître le temps libre et de satisfaire de nouveaux besoins, jouent un rôle très important dans le système productif (Bailly et Maillat, 1988; Barcet, Bonamy et Mayere, 1988). 
Ces auteurs remettent donc en question la vision traditionnelle selon laquelle l'industrie se situe au centre de tout développement. Ils considèrent que la classification de Clarke est dépassée, qu'elle n'a plus de sens dans les économies modernes où on ne peut plus appliquer la notion de résidu aux services. Le tertiaire, loin d'être homogène, serait constitué d'un grand nombre d'activités très diverses. Enfin, il ne serait plus possible de considérer les changements structurels de l'emploi comme si l'industrie et les services fonctionnaient de manière totalement indépendante, puisque, au contraire, ces changements interviennent par le truchement d'une intégration croissante des deux secteurs d'activité, (Jeanneret et al., 1984; Kempf, 1984; Boulianne et Thévoz, 1985; Siniscalco, 1985; Bailly \& Maillat, 1986; Boulianne et Mettan, 1987).

Parce qu'on est, au cours des ans, passé du tertiaire traditionnel - commerces de détail, services d'entretien - à des activités tertiaires qui s'intègrent dans l'industrie et exportent une partie de leurs services - commerce de gros, bureaux de recherche, d'ingénierie, d'assurance, de finance - ces chercheurs proposent de nouvelles classifications de l'emploi qui permettraient de mieux rendre compte de l'imbrication des activités tertiaires et secondaires.

A cet effet, Noyelle (1985) reprend les grandes lignes de la classification de Clarke tout en la complétant. Aux deux catégories d'activités primaires et secondaires, il ajoute 6 classes d'activités de services basées sur la nature du produit ou service et sur son cadre institutionnel. Ces regroupements permettent de classer les activités suivant leur étape dans le processus de production, qu'elle soit intermédiaire ou finale et suivant la nature du client, qu'il soit une entreprise ou un consommateur.

Cette distinction entre les activités de service destinées au consommateur et celles qui s'adressent à l'entreprise fait également l'objet de recherche par Bailly et Maillat (1986). Ces chercheurs proposent une division des activités tertiaires selon qu'elles sont ou non créatrices de richesse. Aux services à la population ou tertiaire banal, ils suggèrent d'ajouter les activités liéesà la production, qu' ils divisent en deux groupes. Le premier, qu'ils définissent comme lié interne, se rapporterait aux activités de services à l'intérieur des entreprises : recherche et développement, gestion, commercialisation, planification. Le second, lié externe, regrouperait les services offerts par les entreprises extérieures : banques, assurances, ingénierie, crédit-bail, expertise en recherche et publicité, auprès desquelles, pour des raisons de rentabilité, de souplesse et d'efficacité, les entreprises achètent souvent des services.

Sur la base de ces premiers travaux, les chercheurs de l'IRER élaborent une typologie des activités de l'emploi qui devrait permettre d'atteindre un nouvel idéal économique (Boulianne et Thévoz 1985; Boulianne et Mettan, 1987; Bailly et Maillat, 1988). Cette nomenclature est composée de quatre types d'activités :1) le secteur de fabrication occupé à la transformation de matières premières; 2) celui de circulation, regroupant toutes les activités intermédiaires reliées au flux des personnes, des biens, des informations, des communications et de la finance; 3 ) celui de 
distribution, dont la tâche est de mettre biens et services à la disposition du consommateur; 4) les activités de régulation qui sont assurées par les établissements intervenant dans le fonctionnement de l'ensemble du système pour le maintenir, le modifier, le réglementer le contrôler. Suivant ce modèle, la répartition idéale de la main d'oeuvre serait de $30 \%$ pour la fabrication, la circulation et la distribution et de $10 \%$ pour la régulation.

Enfin, se basant sur le rôle des services dans l'industrie, Barcet, Bonamy et Mayere (1988) considèrent que le «service productif est en voie de transformation d'une économie de biens à une économie de fonctions et qu'il serait plus approprié de parler de produits-services». Suivant ce concept, les activités de l'entreprise seraient réparties entre cinq fonctions : administration de la production, gestion des facteurs de production, de l'acte de vente, du traitement de l'information et de l'administration générale.

Plutôt que d'évaluer la productivité des services à partir des modèles élaborés pour le secteur manufacturier, on suggère de mettre en place un système de mesure adapté à la spécificité des services (Rabier et Sipek, 1985), ou encore d'en mesurer la productivité par les performances du système productif dans son ensemble.En effet, une étude menée au Etats-Unis par Brender, Chevallier et Pinsani-Ferry (1988) vient confirmer ce fait en faisant ressortir le décalage qui existe entre les deux types d'activités, soit les services d'intégration, transports, commerce de gros, financiers et immobiliers, qui «établissent des réseaux de connexions entre les différentes activités économiques» et les services de distribution qui «assurent à la périphérie du système productif la distribution des biens et services à la population» (p.112).

Se basant sur l'imbrication toujours plus grande entre les activités de production et de service, un certain nombre de chercheurs ont orienté leurs travaux sur la manière dont les services seraient susceptibles de prendre le relai de l'industrie dans l'économie et particulièrement dans le développement régional. Ces initiatives ont pour point de départ les conséquences de la dernière crise économique sur les activités de production des centres industriels.

Dès le début des années 1970, on commence à remettre en question la théorie des lieux centraux en déplorant le gigantisme, la désuétude et le manque de flexibilité des grands centres industriels (Schon et al., 1971). Des critiques similaires sont formuléesàl'endroit des grandesentreprises, traditionnellement leadersdel'économie, mais qui seraient devenues trop complexes et trop rigides pour répondre aux changements d'une manière adéquate (Bailly et Maillat, 1986). A ces anciens modèles, on propose sinon d'en substituer, du moins d'en ajouter de nouveaux dans lesquels le tandem activités de services et PME jouerait un rôle prépondérant.

C'est sur ce postulat que Planque (1982) oppose au système de développement par le haut, reposant sur les centres industriels et sur leur effet d'entrainement dans les régions périphériques, un développement par le bas qui se ferait à partir des 
régions et dans lequel les PME joueraient un rôle stratégique, parce que plus flexibles et plus innovatrices.

Mais, en raison de leur petite taille et de leurs ressources limitées, elles n'ont pas les moyens d'internaliser un certain nombre de tâches nécessaires aux activités de production. Il est donc indispensable qu'elle puissent compter sur une infrastructure de services : transports, services de main d'oeuvre, de génie, d'entretien, de construction, programmes d'appui technologique, services financiers (Aydalot, 1979; Planque, 1982; Boulianne, Maillat et Rey, 1983; Bailly et Maillat, 1986).

Pottier (1984) dit très justement que les conditions du développement régional sont une combinaison de facteurs : des activités de recherche et de formation; un tissu industriel de départ et des activités structurées, des relations entre les PME et les grandes entreprises; enfin un état d'esprit qui favorise cette mise en place. En d'autres mots, une infrastructure adéquate.

Ainsi, les activités de services, loin d'être un fardeau pour l'économie, seraient appelées à jouer un rôle important dans la prospérité des entreprises, particulièrement dans celles des villes moyennes et décentralisées. Les auteurs y mettent cependant certaines conditions : il faut que le contrôle de l'infrastructure soit et demeure régional; il faut que l'éloignement des régions développées ne soit pas trop grand; enfin, la région doit voir la fin de la mono-industrie et l'introduction des activités tertiaires mettant l'accent sur l'identité régionale et le développement de structures d'accueil (Aydalot, 1984; Bailly, Maillat et Rey 1983).

Dans cette optique, nous avons cherché à savoir à quelle étape de ce processus se situe le Nord-Est de l'Ontario et comment y est perçu le rôle des services dans le développement économique régional.

\section{Les services en région périphérique : analyse des perceptions}

Le Nord-Est de l'Ontario ${ }^{2}$ fait partie de l'hinterland; c'est une région immense, peu peuplée et relativement éloignée des centres industriels et administratifs. Sa base économique, le plus souvent de caractère mono-industriel, repose sur l'exploitation et la semi-transformation des richesses naturelles : bois et produits miniers. La dernière récession, la modernisation et la rationalisation des installations qui ont suivi ont eu pour conséquence une baisse importante des effectifs industriels que n'a pu combler totalement la création d'emplois dans les services, puisqu'entre 1981 et 1986, la population du Nord-Est a connu une diminution de 2,5\%.

2 Le Nord-Est de l'Ontario est formé par la vaste région située au nord de la Rivière-desFrançais entre le Québec et le Lac Supérieur. Ce territoire compte pour $30 \%$ de la superficie de l'Ontario et environ $6 \%$ de sa population. 
La transition vers une prédominance de l'emploi dans le secteur tertiaire s'y est manifestée beaucoup plus tardivement que dans les régions centrales, mais s'y est accélérée depuis quinze ans, de sorte que la région se compare aujourd'hui aux modèles provincial et national (Statistique, Canada, 1951 à 1986).

\section{1 Échantillon et questionnaire}

La population étudiée était composée de gens d'affaires francophones des villes de Sudbury, North Bay, Timmins, Sturgeon Falls et Noelville ${ }^{3}$. Pourquoi une étude auprès de la population française dans une région largement anglophone? Pour plusieurs raisons. Les francophones constituent une proportion importante de la population dans la plupart des districts du nord-est de l'Ontario. Cette proportion va de $28 \%$ dans le Timiskaming à $33 \%$ à Sudbury et à $51 \%$ dans le district de Cochrane (ACFO, 1988). Elle est essentiellement française dans certains secteurs du Nipissing comme les localités de Sturgeon Falls et de Noelville. Les auteurs sont généralement d'accord sur le fait que le marché canadien-français englobe les neuf comtés limitrophes de l'est et du nord-est de l'Ontario (Brisoux, Darmon, Laroche, 1987). Enfin, l'importance du français s'est considérablement accrue depuis l'entrée en vigueur de la loi fédérale sur les langues officielles et particulièrement depuis l'établissement des services en français mis de l'avant par le gouvernement provincial.

Pour nous permettre de recueillir les données nécessaires à l'analyse, 769 questionnaires furent envoyés. Cet envoi fut suivi d'un rappel par téléphone. La population interrogée était composée de 113 personnes provenant de la fonction publique, 90 du secteur manufacturier et 566 des autres services; toutes possédaient au minimum un diplôme universitaire de premier cycle. Parmi les 769 questionnaires envoyés, 183 ont été retournés, soit un taux de réponse de $23,7 \%$, ce qui est excellent en regard du taux habituel de $10 \%$ que l'on rencontre dans les enquêtes par la poste. On retrouve en quelque sorte une sur-représentation du secteur des services surtout par rapport au domaine manufacturier. Ceci reflète toutefois la composition de la population des affaires, car l'industrie du nord-est se caractérise par un petit nombre de très grandes compagnies axées sur l'exploitation des richesses naturelles, et d'une multitude de petites compagnies.

Les caractéristiques socio-économiques de la population interrogée sont rapportées au tableau 1, p. 354.

3 Toutes ces personnes figurent dans la $2^{c}$ édition du Répertoire des gens d'affaires francophones de Nordcom, une organisation à but non lucratif dont l'objectif est de promouvoir les affaires et la formation des cadres parmi la population francophone du Nord-Est de 1'Ontario. 
Tab. 1 Caractéristlques socio-économiques nord-ontariens et de leur compagnie

Secteurs d'industrie

a) gouvernement

$20,7 \%$

b) services

$68,7 \%$

c) secteur manufacturier

$10,6 \%$

Occupation des répondants
a) directeurs
b) propriétaires
c) gérants/superviseurs
d) professionnels/agents/vendeurs
e) autres

$23,1 \%$

Taille des compagnies
tp) très petites
p) petites
m) moyennes
g) grandes
tg) très grandes

1 - 5 employés

$26,0 \%$

6- 15 employés

$33,8 \%$

16 - 50 employés

$22,5 \%$

51 - 199 employés

$9,3 \%$

200 employés et plus

$8,5 \%$

Age des compagnies
tj) très jeunes
$1-5$ ans
$13,7 \%$
j) jeunes
$6-10$ ans
$16,5 \%$
m) moyennes
$11-25$ ans
$33,1 \%$
v) vieilles
26 ans et plus
$36,7 \%$

Types de compagnies
a) société par actions
b) franchises
c) PME
d) gouvernement
e) coopératives
f) autres

$17,3 \%$

$38,0 \%$

$21,8 \%$

$7,3 \%$

$9,5 \%$

Le regroupement des compagnies selon la taille de l'entreprise ou l'importance de la main-d'oeuvre ne respecte pas les critères généralement acceptés dans la définition des PME. Il nous paraît cependant mieux approprié au contexte de cette étude, puisque rares sont les entreprises qui comptent plus de 500 employés.

Le but de l'étude était de connaître les opinions des répondants sur l'importance qu'occupe actuellement la région dans le monde des affaires, sur le niveau de croissance économique dans le secteur d'activité de chaque répondant, sur leurs prévisions quant au développement économique futur de la région, enfin sur leurs opinions quant aux secteurs qu'ils prévoyaient être les futurs générateurs d'emploi, 
à savoir les manufactures, les mines, les forêts, la fonction publique, le tourisme, la finance, la vente au détail, la restauration et le transport. Une sous-question visait à découvrir jusqu'à quel point le nord-ontarien se montre favorable aux gens d'affaires francophones. Enfin une question ouverte portait sur les mesures suggérées pour maintenir et/ou améliorer le développement de l'économie régionale 4 .

\subsection{Méthode d'analyse}

Les données étant de type qualitatif, il fallait trouver une méthode d'analyse qui permette de les traiter de manière adéquate. C'est pourquoi il fut décidé d'utiliser la méthode d'analyse de correspondance multiple(ACM), une technique multivariée qui permet de présenter graphiquement les relations existant entre plus de deux variables qualitatives.

Cette méthode a été développée par Benzecri (1972) et Lebart et Tabart (1973) sur la base des travaux antérieurs de Burt (1950). Plusieurs livres et articles ont par la suite été publiés sur le sujet (Benzecri, 1973; Nashisato, 1980; Gifi, 1981; Greenacre, 1984; Lebart, Morineau et Warwick 1984; Hoffman et Franke, 1986; Green, Krieger et Carroll, 1987; Carroll et Green, 1988, Carroll, Green et Schaffer, 1986, 1987), augmentant par le fait même l'intérêt des chercheurs nord-américains pour cette méthode. Le rapport qui existe entre l'ACM et les autres méthodes d'analyse qualitative multivariée a été analysé par Tenenhaus et Young (1985).

Les données analysées par cette méthode ont été reportées au moyen d'un tableau de description dans lequel les lignes correspondaient aux sujets, c'est-à-dire aux opinions face à l'importance de la région dans le monde des affaires, au taux de croissance économique du secteur spécifique d'activités de chaque répondant, aux prévisions de développement et aux possibilités offertes aux gens d'affaires francophones. Pour leur part, les colonnes correspondaient aux catégories de réponses, à savoir «beaucoup, assez et insuffisant»; cette dernière catégorie regroupait les catégories «très peu» et "pas du tout» qui, prises individuellement, ne recueillaient pas suffisamment de suffrages pour l'analyse. A la question concernant les prévisions économiques, les catégories étaient «excellent, stable et en déclin/indécis».

Les caractéristiques socio-économiques des répondants et des compagnies, soit les secteurs d'industrie, l'occupation des répondants, la taille, l'âge et le type de leur compagnie, de même que les secteurs futurs générateurs d'emplois ont ensuite été traitées comme variables supplémentaires.

Grâce à l'utilisation de l'ACM, il fut possible d'analyser toutes ces variables simultanément pour ensuite établir les relations qui existaient entre elles. Il està noter que les dix questions sont hétérogènes, c'est-à-dire qu'elles comportent toutes un nombre différent de catégories de réponses. Par exemple, on en retrouve 3 à la question 1 ( $a, b$ et c) et 5 à la question $2(a, b, c, d, e t e)$. Si toutes comportaient le même

$4 \quad$ L'annexe l présente le questionnaire partiel utilisé pour la cueillette des données. 
nombre de catégories, il serait possible de les soumettre directement à l'ACM au moyen du tableau de description $\mathrm{Z}$. Tel n'étant pas le cas, il a fallu procéder à la pondération des dix questions afin de neutraliser les facteurs qui auraient pu apparaitre uniquement parce que le nombre de catégories de réponses différait suivant les questions (Greenacre, 1984, p. 162). Le mode de pondération des variables dépend de leur inertie. Si le nombre de catégories de réponses de la variable JK est $\mathrm{jK}$, son inertie $\mathrm{In}(\mathrm{jK})$ peut être calculée de la manière suivante :

$$
\ln (\mathrm{j} K)=(\mathrm{jk}-1) / \mathrm{p},
$$

où p représente le nombre total de variables (Greenacre, 1984, p. 139). Ainsi, In(JI), In(J2), ...In(JP) indiquent les inerties des variables $\mathrm{Jl}, \mathrm{J} 2, \ldots . \mathrm{Jp}$, respectivement. Il $s$ 'agit ensuite de pondérer les variables afin d'égaliser leurs inerties en multipliant les colonnes correspondant aux variables du tableau $Z$ par les facteurs $v 1, v 2, \ldots v p$, afin que v1In(j1) = v2In $(J 2)=v p I n(j p)=v$ où v est une constante (Greenacre, 1984, p. 167). Dans ce cas, $p=10$ et les inerties des dix variables sont :

$$
\begin{aligned}
& \ln (1)=2 / 10, \ln (2)=4 / 10, \ln (3)=4 / 10, \ln (4)=3 / 10, \ln (5)=5 / 10, \\
& \ln (6)=2 / 10, \ln (15)=9 / 10, \ln (6)=\ln (7)=\ln (8)=\ln (13)=2 / 10 .
\end{aligned}
$$

En choisissant $v=2$, il est possible de neutraliser les inerties de ces variables en multipliant les trois premières colonnes du tableau $\mathrm{Z}$, c'est-à-dire les catégories de réponses de la variable $Q 1$ par le coefficient $v 1+10$, puis les cinq colonnes suivantes du tableau $Z$, c'est-à-dire les catégories de réponses de la variable $Q 2$ par le facteur de pondération v2 $=30 / 4$ et ainsi de suite, pour définir le tableau de description $Z$ transformé en RZ. On peut ensuite utiliser directement cette matrice pondérée dans l'analyse. Pour une description de l'algorythme de l'ACM on peut se référer, entre autres, à Greenacre (1984, ch. 5).

\subsection{Résultats}

Avant de passer à l'analyse des données traitées par la méthode de correspondance multiple, nous avons jugé bon de procéder à une distribution des fréquences des variables d'opinions. Ces résultats préliminaires sont rapportés dans le tableau 2.

Ces résultats, basés sur le calcul des simples pourcentages, nous indiquent que, de manière générale, les personnes interrogées tendent à se montrer relativement optimistes face à la santé de l'économie de la région, puisque la très grande majorité des répondants émettent des opinions qui, à toutes les questions du tableau ci-dessus, se situent sur l'axe positif, c'est-à-dire dans les catégories «beaucoup» et «assez». Ces opinions regroupées vont de 73,5\% pour l'importance qu'aurait prise le NordOntarien dans le monde des affaires à $92,4 \%$ quant à ses perspectives futures de développement.

C'est la catégorie «assez» qui regroupe le plus de suffrages avec $43,7 \%$ à la question 6 (importance de la région quant à sa position dans le monde des affaires), 
$44,9 \%$ à la question 7 (croissance économique dans le secteur des répondants) et 51,9 $\%$ à la question 8 (opportunités offertes aux gens d'affaires francophones). Son correspondant «stable» à la question 13 (perspectives de développement économique) recueille $49,7 \%$ des suffrages.

Tab. 2 Opinions des gens d'affaires francophones vis-à-vis de l'économie nord-ontarienne

\begin{tabular}{|lcccc|}
\hline Opinions & Beaucoup & Assez & Très peu & $\begin{array}{c}\text { Pas du } \\
\text { tout }\end{array}$ \\
\hline $\begin{array}{l}\text { Croyez-vous que le nord-ontarien } \\
\text { a pris de l'importance quant à sa } \\
\text { position dans le monde des } \\
\text { affaires ? (Q.6) }\end{array}$ & $27,9 \%$ & $43,7 \%$ & $26,8 \%$ & $1,6 \%$ \\
$\begin{array}{l}\text { Dans quelle mesure voyez-vous } \\
\text { une croissance économique } \\
\text { dans votre secteur ? (Q.7) }\end{array}$ & $30,6 \%$ & $44,8 \%$ & $22,9 \%$ & $1,7 \%$ \\
$\begin{array}{l}\text { Jusqu'à quel point le nord-ontarien } \\
\text { est-il favorable aux gens d'affaires } \\
\text { francophones ? (Q.8) }\end{array}$ & $29,0 \%$ & $51,9 \%$ & $18,0 \%$ & $1,1 \%$ \\
\hline & Excellent & Stable & En déclin & Indécis \\
\hline $\begin{array}{l}\text { Dans le futur, comment voyez- } \\
\text { vous le développement }\end{array}$ & $43,7 \%$ & $49,7 \%$ & $5,3 \%$ & $2,1 \%$ \\
\hline économique du nord ? (Q.13) & & & & \\
\hline
\end{tabular}

Dans un deuxième temps, nous avons cherché à savoir comment se répartissent ces opinions si on les met en corrélation avec l'occupation des répondants et avec les caractéristiques socio-culturelles de leur compagnie. L'ACM nous permet d'y répondre et les résultats sont présentés à la figure 1, p. 359.

Puisque les deux axes principaux qui définissent la figure 1 permettent d'expliquer $74,26 \%$ de la variance totale, il fut décidé de limiter l'analyse à ces deux premiers axes. L'étude de cette figure révèle que les trois catégories de réponses de chaque variable d'attitudes prennent la forme d'une courbe parabolique concave. Ces variables d'attitudes sont l'importance prise par le nord-ontarien quant à sa position dans le monde des affaires (6), la croissance économique dans le secteur d'activités des répondants (7), opportunités offertes aux gens d'affaires francophones (8), enfin, les prévisions quant au développement économique futur du nord-est ontarien (13).

Ainsi, toutes les catégories «a» (beaucoup) des quatre questions se retrouvent localisées dans la section inférieure gauche de la courbe. De même, toutes les catégories «c» (très peu/pas du tout) sont situćes dans la partie inférieure droite, et 
toutes les réponses «b» (assez) sont concentrées au sommet de la courbe. Cette distinction signifie qu'il est possible de diviser les répondants en trois segments : les optimistes, ceux qui ont répondu «a» à toutes les questions, les pessimistes, ceux qui ont répondu «c» à toutes les questions, et les modérés ou indécis, ceux qui ont répondu «b» à toutes les questionss.

Si l'on relie aux segments décrits plus haut les variables supplémentaires suivantes: le secteur d'activités (l), l'occupation des répondants (2), la taille (3), l'âge (4), et le type de leur compagnie (5), ainsi que les secteurs d'industrie futurs générateurs d'emplois (15), on constate plusieurs faits intéressants.

A un extrême, on retrouve les optimistes, c'est-à-dire ceux qui se montrent extrêmement satisfaits de la croissance économique qui s'est manifestée dans leur secteur d'industrie. Cet optimisme leur donne une grande confiance face au développement futur de la région et aux opportunités offertes aux francophones.

Ils proviennent du secteur des services (1b) où ils sont propriétaires (2b) ou professionnels/agents dans l'immobilier, les services financiers, comptables, communautaires et personnels (2d), de petites (3p-6-15 employés) et très jeunes compagnies (4 tj -1-5 ans), qu'elles soient des franchises ( $5 b$ ), des PME (5c) ou des sociétés par actions (5a). Les services financiers (15f), les transports (15i) et le secteur manufacturier $(15 \mathrm{~h})$ leurapparaissent comme les principaux futurs générateurs d'emplois.

Ce sont ceux qui retirent le plus grand bénéfice deschangementséconomiques qui se montrent les plus optimistes. Cette bonne fortune sourit aux petites compagnies établies depuis 1983, à la faveur de la reprise économique qui a suivi la crise des années 1981 et 1982 et de l'explosion des services qui l'a caractérisée. Il faut certainement y voir un effet de la saturation des services, et particulièrement des franchises, dans les grandes villes du sud, d'où expansion vers les régions périphériques. On retrouve donc ici, pour reprendre la théorie de Planque (1982), le modèle de développement par le haut.

Cet optimisme est encore motivé par l'usage accru et le statut du français dans la région. A la faveur des initiatives gouvernementales favorisant l'extension du bilinguisme comme la loi fédérale sur les langues officielles ou les programmes provinciaux, le français acquiert peu à peu ses lettres de noblesse et l'industrie des services, plus que tout autre, a toutes les raisons de s'en réjouir.

Enfin, il est un fait que durant les périodes de changement de la structure industrielle, les petites entreprises connaissent beaucoup plus de succès que les grandes organisations en raison de leur plus grande flexibilité. L'épanouissement de

5 La suite du questionnaire portait sur les opinions vis-à-vis de la gestion de l'entreprise, de la formation des gestionnaires et administrateurs, et sur les opportunités offertes aux personnes bilingues. Ces domaines feront l'objet d'analyse ultérieure car ils dépassent l'objectif du présent article. 
Fig. 1 L'économie nord-ontarienne : analyse des perceptions par la méthode de correspondance multiple

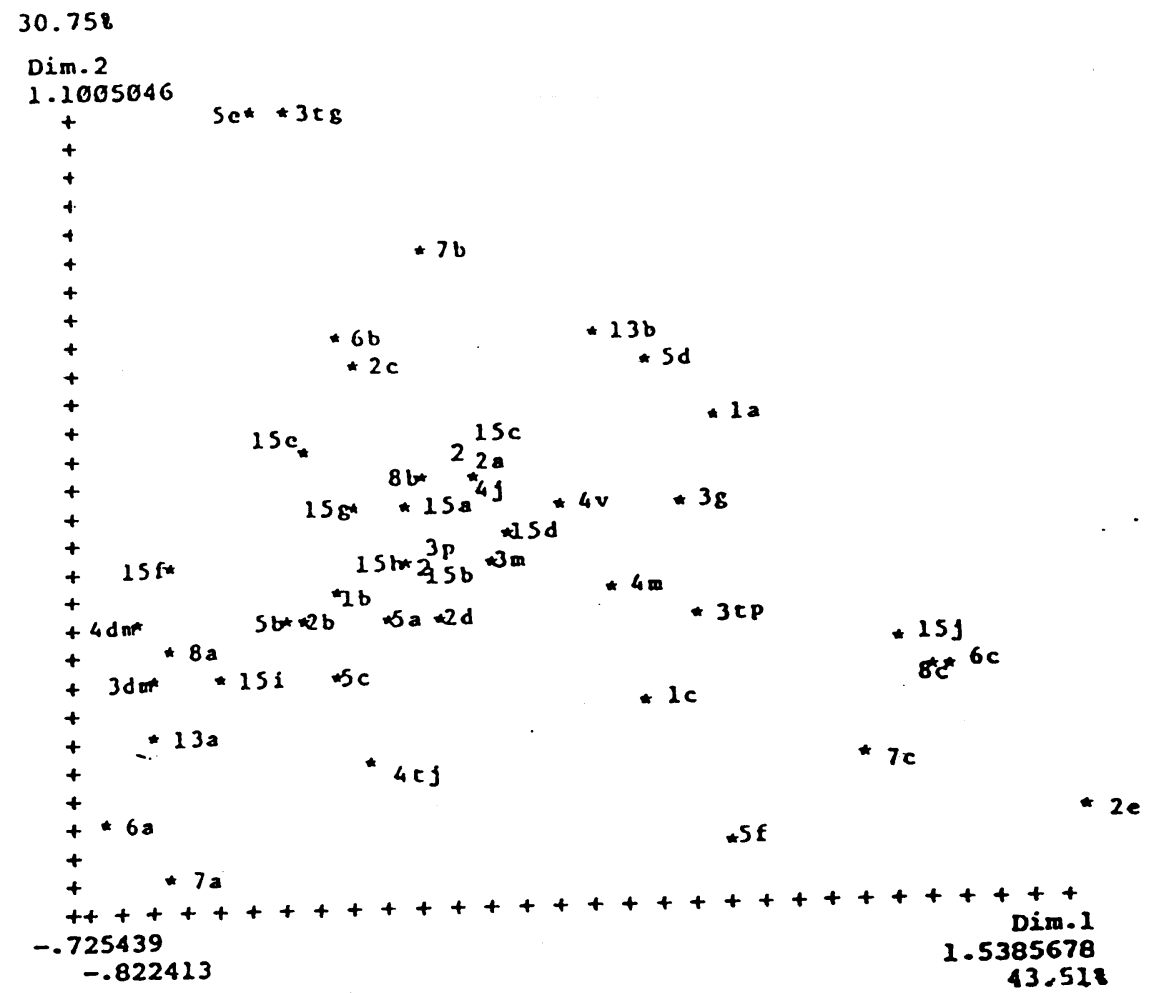

DONNEES DE TETES POUR : AXI12

NOMBRE DE CASES $\quad: 46$

NOMBRE DE VARIABLES : 2 
ce type de compagnie peut encore s'expliquer par le fait que ces entrepreneurs ont su percevoir les opportunités qui se présentaient et en profiter.

A l'autre extrême, se retrouvent les pessimistes. Ce sont soit des propriétaires de très petites entreprises (3tp-1-5 employés) du secteur manufacturier, soit des directeurs (2a) de services importants dans la fonction publique (1a; $3 g-51-199$ employés). Dans un cas comme dans l'autre, ces entités sont établies depuis plus de 10 ans ( $4 m-11-25$ ans; $4 v-26$ ans et + ).

Le secteur manufacturier ayantété le plus affecté par la crise économique et par les changements économiques intervenus depuis deux décennies, on peut s'attendre à retrouver un certain pessimisme dans ses rangs. L'âge de ces compagnies vient encore confirmer ces tendances. Mais il y a plus. En raison de l'éloignement des marchés, de la faible densité de population, de l'absence d'infrastructure de services et de voies de communications adéquates, on ne retrouve dans la région pratiquement aucune industrie orientée vers les technologies de pointe (Gillingham \& Ribordy, 1988). Ces compagnies sont axées avant tout sur des activités de support aux industries minières et forestières pour leurs besoins en acier, en machines de chantier et en matériel de construction. Dans ce contexte de mono-industrie, «les grandes compagnies déterminent les règles du jeu et lesPME sont soumises aux contingences de la réalitééconomique» (d'Amboise, 1989, p. 33). Il n'est donc pas surprenant de constater qu'elles se montrent pessimistes dans une période de bouleversements industriels.

La même tendance se retrouve encore dans les réponses à la question ouverte où on réclame le développement d'une industrie secondaire plus importante et une assistance accrue pour y parvenir; on désire aussi briser l'isolement ressenti face aux centres nerveux de la province puisqu'on revendique la construction d'autoroutes à quatre voies et un meilleur système de communication. Ce sont des préoccupations que l'on retrouve dans les recommandations du Conseil du Premier Ministre (1988): depuis le début des années 1980, «certaines régions de la province, surtout celles du Nord et de l'Est, n'ont eu qu'une participation réduite à l'expansion économique» (p. 11).

Ce pessimisme se retrouve parmi les membres de la fonction publique dont l'expansion dans la région fut importante depuis quinze ans en raison de la décentralisation et de la relocalisation des ministères fédéraux et provinciaux. Jusqu'à quel point cette décentralisation joue-t-elle un rôle sur les opinions exprimées ? Faut-il y voir le manque de flexibilité d'une immense machine ou un sentiment de frustration motivé par l'obligation d'avoir dû quitter les grands centres contre son gré pour s'installer dans une région périphérique n'offrant pas toutes les commodités auxquelles on est habitué ? Où est-ce le fait que les gouvernements se mettent à décentraliser leurs services durant les périodes économiques difficiles ? Ces opinions nous révèlent peut-être une vision plus globale de l'économie face a un certain déclin des industries.

En dernier lieu, les modérés ou indécis se retrouvent soit parmi les superviseurs (2c) de très grandes entreprises (3tg - 201 employés et + ), soit parmi les gérants (2c) 
de coopératives (5e). Les coopératives sont représentées ici par les caisses populaires dont le nombre est relativement important dans le nord-est de l'Ontario. La vente au détail leur apparaît comme celle offrant le plus d'emplois dans le futur (15e).

On retrouve dans ce groupe les gérants de coopératives, c'est-à-dire d'organismes qui peuvent être considérés comme des PME en raison de leur taille. Les attitudes de ces personnes présentent de grandes similitudes avec les caractéristiques de ceux que d'Amboise (1989) définit comme les dirigeants d'entreprises alternatives, axés sur les exigences administratives qui «travaillent de longues heures, mais ils le font surtout pour des projets qui les enthousiasment sans trop se soucier du long terme, [...] Comme les exigences administratives sont souvent pour eux des contraintes, ils vivent difficilement les transitions nécessaires dans l'évolution de la petite et moyenne organisation qu'est la coopérative»(p. 102-3).

L'attitude des modérés, ou plutôt des indécis, porte à penser qu'ils ne tiennent pas à se prononcer dans ce débat par souci de protéger une situation de neutralité; ou parce que se trouvant entre les deux extrêmes, ils perçoivent le déclin d'un modèle économique et l'émergence de l'autre; ou qu'enfin, il leur est difficile de porter un jugement sur un développement qui les dépasse. Cette attitude se reflète dans leurs prévisions quant au secteur futur générateur d'emploi, car la vente au détail a certes connu une croissance depuis les années 1960, mais cette augmentation n'a aucune commune mesure avec celle des autres services; et surtout, c'est un secteur qui n'est nullement créateur de richesse.

A la question ouverte portant sur les mesures recommandées pour améliorer l'économie de la région, on préconise tout d'abord le développement du secteur manufacturier, d'innovations, du tourisme, et une plus grande décentralisation de la fonction publique. Viennent ensuite des préoccupations relatives aux barrières de communications avec le sud de la province et à la promotion économique de la région, c'est-à-dire à des mesures visant à briser l'isolement dans lequel on se perçoit. En troisième lieu, on aimerait voir une aide gouvernementale à la petite entreprise plus importante de même qu'une réduction des impôts et de la paperasse. L'amélioration des moyens de communication, comme la construction d'autoroutes à quatre voies pour relier les régions industrielles et administratives du sud de la province viennent en dernier.

\section{Conclusion}

L'analyse des changements économiques qui se sont manifestés dans le Nord-Est de l'Ontario depuis deux décennies et leur perception par les gens d'affaires a révélé certains faits très intéressants. Lorsque comparé aux régions de tradition industrielle, le Nord-Est de l'Ontario présente un modèle particulier et spécifique à l'hinterland, largement décentralisé, axé sur la mono-industrie d'exploitation et de transformation sommaire des richesses naturelles. 
Le concept de centralité-marginalité dont parle Bailly (1983) s'y applique sans aucun doute tant il est vrai que la région est soumise à des relations dissymétriques sur les plans politique, économique et culturel. Cette réalité est très bien mise en lumière dans les réponses à la question ouverte puisque les membres de la communauté des affaires déplorent l'éloignement géographique des marchés et des centres de décision, l'absence d'infrastructureéconomique et de services etl' isolement psychologique.

On y retrouve, comme partout ailleurs, un recul, plus tardif mais plus accéléré, de la proportion du secteur industriel dans l'économie, et par voie de conséquence, un recul des salaires et de la productivité générale. Si, comme l'affirment les membres du Conseil du Premier Ministre (1988), la prospérité d'un pays est directement proportionnelle à sa capacité de création de richesse dans les secteurs exportateurs, on peut parler de déclin macro-économique, car, depuis le début des années 80 , cette région n'a eu qu'une participation restreinte au développement économique (p. 11). C'est aussi la perception qu'en ont les propriétaires de petites entreprises du secteur manufacturier, les directeurs de très grandes compagnies et les membres de la fonction publique.

$\mathrm{Si}$, par contre on examine l'économie d'un point de vue micro-économique, on note un essor considérable du secteur des services, puisque d'un tiers qu'elle était durant les années 1950, la proportion de la main-d'oeuvre employée dans les services est passée à près de $70 \%$ en 1986. L'analyse a révélé l'émergence d'une nouvelle classe de gens d'affaires, celle des propriétaires et gestionnaires de franchises et de PME des services qui, durant une période de changements industriels, ont su percevoir et saisir les opportunités qui se présentaient. D'une part, ils profitent en quelque sorte de la marginalité résultant des changements structuraux de l'économie en exploitant, dans des commerces de détail et de distribution, des employés souspayés. D'autre part, ils participent à la création de richesse en offrant des services financiers et comptables aux entreprises.

Cette croissance sans précédent des PME de services est-elle un phénomène passager, une étoile filante ? Au contraire, il est permis d'en douter puisque la part des services dans l'économie devrait encore s'accroître durant la prochaine décennie et avec elle, le nombre et le type d'entreprises du secteur tertiaire, et particulièrement celles qui sont axées sur la distribution des biens et services, tandis qu'on assiste à la diminution de la mono-industrie et l'introduction d'activités tertiaires.

On y retrouveraaussi un nombre croissant de franchises. Or, peut-on considérer ce type d'entreprise comme étant une PME ? Cela ne fait aucun doute si l'on se réfere à sa taille, mais le consensus est loin d'exister sur la question de son idéologie. Il y a certainement lieu d'assimiler le gestionnaire de ce type de compagnie à celui que Knight (1981) définit comme le troisième type d'entrepreneur, celui du directeur général qui fait fonctionner sa compagnie comme une entreprise commerciale. Il a sans aucun doute une mentalité d'entrepreneur en sachant percevoir les occasions d'affaires et en mettant en place les ressources nécessaires à la réalisation de son projet. 
Peut-on, en déclarant que les services contiennent peu de potentiel de croissance qui ne soit pas une demande dérivée de la production des biens parler de déclin ? Devrait-on plutôt parler de transformation? Puisque la transition de l'emploi vers le secteur tertiaire s'y est faite beaucoup plus tardivement que dans les régions centrales, mais d'une manière sensiblement plus accélérée, la nature de ce changement doit, par le fait même, être différente. Quelles sont les étapes de ce développement ? Devrait-on passer en premier lieu par l'étape de service à la population. C'est ce que suggère Aydalot (1984) pour qui, entre la mono-industrie et les activités de production diversifiées, doivent intervenir des activités essentiellement tertiaires de service à la population, comme le tourisme et les loisirs, afin de rendre la région plus attrayante et d'y attirer des cadres d'entreprises.

Si c'est là une étape nécessaire dans les changements économiques, les PME des services sont certainement de nouvelles vedettes en ce sens qu'elles pourraient bien être les précurseurs d'un nouvel ordre économique en région périphérique. Afin de répondre de manière plus définie à cette question, il conviendrait de répéter cette expérience sur d'autres régions périphériques axées sur l'exploitation des richesses naturelles ou encore d'observer l'effet des changements structurels de l'emploi sur une période plus longue.

\section{BIBLIOGRAPHIE}

A.C.F.O., (1988), Les francophones tels qu'ils sont en 1986. Recueil statistique, Vanier, Association Canadienne Française de l'Ontario.

Amboise (d'), G., (1989), La P.M.E. canadienne, situation et défi, Québec, les Presses de l'Université Laval.

Aydalot, Ph., (1984), «Le dynamisme économique a-t-il déserté la ville?», Paris, Dossiers du Centre Economie, Espace, Environnement, $n^{\circ} 39$.

Aydalot, Ph., (1979), «Contribution à l'analyse de la division spatiale du travail», Paris VII, Dossiers du Centre Economie, Espace, Environnement.

Bailly, A.S.et Maillat, D., (1986), «Activités de service et développement régional», Neuchatel, IRER, Université de Neuchatel.

Bailly, A.S. et Maillat, D., (1988), Le secteur tertiaire en question, Paris, Economica, $2^{\mathrm{e}}$ édition.

Bailly, A.S., (1983), «La marginalité : réflexions conceptuelles et perspectives en géographie, sociologie et économie», Géotopiques, Universités Lausanne-Genève, p. 73-113.

Bandt (de), J., (1985), «Connaissance et méconnaissance des activités de service», dans J. de Bandt (éd.), Les services dans les sociétés industrielles, Paris, Economica, p. 201-223.

Barcet, A. et Bonamy, J., (1985), «Services rendus aux entreprises et dynamique du système productif», dans J. de Bandt (ed.), Les services dans les sociétés industrielles, Paris, Economica, p. 107-137.

Barcet, A., Bonamyet, J. et Mayere, A., (1988), «Les services aux entreprises : problèmes théoriques et méthodologiques», dans A.S. Bailly et Maillat, D., (éd.), Le secteur tertiaire en question, Paris, Economica, p. 118-123.

Benzecri, J.P., (1972), «Sur l'analyse des tableaux binaires associés à une correspondance multiple», Note Mimeo. Lab. Stat. Math., Université Pierre et Marie Curie, Paris. 
Benzecri, J.P., (1973), L'analyse des données, vol. 1 et 2, Paris, Dunod.

Boulianne, L.M. et Thévoz, L., (1985), Nouvelle nomenclature du système de production et rôle des activités de service, Lausanne, C.E.A.T.

Boulianne, L.M. et Mettan, N., (1987), «Nouvelles articulations des systèmes de production et rôle des services : une analyse comparative internationale et inter-régionale», Lausanne, C.E.A.T.

Boulianne, L.M., Maillat, D. et Rey, M., (1983), «L'appui technologique des P.M.E. au niveau national», Rapport final, Lausanne, C.E.A.T.

Brender, A., Chevallier, J. et Pisani-Ferry, J., (1988), «La mouvance de la frontière entre secteur tertiaire et secteur manufacturier», dans A.S. Bailly et D. Maillat (éd.), Le secteur tertiaire en question, Paris, Economica, p. 111-114.

Brisoux, J.E., Darmon, R.Y.et Laroche, M.,(1987), Gestion de la publicité, Montréal, McGrawHill.

Burt, C., (1950), «The Factorial Analysis of Qualitative Data», British Journal of Psychology, (Statistical Section), vol. 3, p. 166-85.

C.L.M.P.C., (1988), «The Growth of the Service Sector and Implications for the Labour Market Productivity», Quarterly Labour Market and Productivity Review, vol. 24, p. 17-24.

Carroll, D.J. et Green, P.E., (1988), «An INDSCAL-Based Approach to Multiple Correspondence Analysis», Journal of Marketing Research, vol. 25, p. 193-203.

Carroll, D.J., Green, P.E. et Schaffer, C.M., (1986), «Interpoint Distance Comparison dans Correspondence Analysis», Journal of Marketing Research, vol. 23, p. 271-80.

Carroll, DJ., Green, P.E. et Schaffer, C.M., (1987), «Comparing Interpoint Distances dans Correspondence Analysis : a Clarification», Journal of Marketing Research, vol. 25, p. 445-50.

Chand, R., (1983), «Why the Dramatic Increase dans Service Sector Employment?», The Canadian Business Review, Autumn, p. 25-28.

Chand, R., (1986), «Employment During the Recession, The Boost From the Services», Canadian Business Review, Summer, p. 37-40.

Christaller, W., (1933), Die Zentralen Orte in Süddeutschland, Jena, Fischer.

Clarke, C., (1951), The Condition of Economic Progress, London, MacMillan.

Conseil du Premier Ministre, Ontario, (1988), Concurrencer dans la nouvelle économie globale, Toronto, Imprimeur de la Reine.

Cunha, A. et Racine, J.B., (1984), «Le rôle des services aux entreprises dans une société postindustrielle : technologies nouvelles et décentralisation», Revue d' Economie Régionale et Urbaine, $\mathrm{n}^{\circ}$ 5, p. 731-755.

Daniels, P., (1978), «Service Sector, Office Employment and Regional Imbalance in Britain», Tijdschrift voor Economische en Social Geographie, n 69, p. 286-295.

Gifi, A., (1981), Non-Linear Multivariate Analysis, Leiden, Dept. of Datatheory.

Gillingham D.W. et Ribordy, A., (1988), «The Influence of Adverse Environmental Conditions on New Product Development», Sudbury, School of Commerce, Laurentian University.

Green, P.E.,Krieger, A.M. et Carroll, D.J., (1987), «Multidimensional Scaling : A Complementary Approach», Journal of Advertising Research, Octobre/Novembre, p. 21-7.

Greenacre, M.J., (1984), Theory and Applications of Correspondence Analysis, London, Academic Press, Inc.

Halami, S., (1989), «Mais qui donc finance la création de millions d'emplois aux Etats-Unis?» Le Monde Diplomatique, mars, p. 6-7.

Hoffman, L. et Franke, G.R., (1986), «Correspondence Analysis : Graphical Representation of Categorical Data in Marketing Research", Journal of Marketing Research, vol. 23, p. 213-27. 
Jeanneret, Ph., Hussy, J., Bailly, A., Maillat, D. et Rey, M., (1984), «Le tertiaire moteur dans les petites et moyennes villes en Suisse : le cas d'Aigle et de Delémont», Lausanne, C.E.A.T.

Kempf, H., (1974), «Croissance des services et réaménagement du secteur productif», Problèmes économiques No 1882, Paris, La Documentation Française, p. 14-19.

Knight, R.M., (1981), Small Business Management in Canada :Text and Cases, Toronto, McGraw-Hill Ryerson.

Lebart, L. et Tabart, N., (1973), «Recherches sur la description automatique des données socio-économiques», Rapport CORDES-CREDOC, Paris.

Lebart, L., Morineau, A.etWarwick, K.M., (1984),Multivariate DescriptiveStatisticalAnalysis, New York, John Wiley \& Sons.

Maillat, D., (1986), «Les P.M.E. innovatrices et la revitalisation des régions de tradition industrielle», Neuchatel, Dossiers de l'IRER, Université de Neuchatel.

Nashisato, S., (1980), Analysis of Categorical Data : Dual Scaling and Its Applications, Toronto, University of Toronto Press.

Noyelle, T.J., (1985), «Dualisme dans les services : le cas des Etats-Unis», dans J. de Bandt (ed.), Les services dans les sociétés industrielles, Paris, Economica, p. 61-83.

Petit, P., (1985), «Les services: un secteur abrité dans la crise», dans J. de Bandt(éd.), Les services dans les sociétés industrielles, Paris, Economica, p. 21-58.

Philippe, J. et Monnoyer, M.C., (1985), L'interaction entre les prestations de services et les PMI et le développement régional, Aix-en-Provence, Centre d'Économie Régionale.

Phillipe, J., (1988), «Les services aux entreprises et la politique de développement régionale», dans Bailly \& Maillat, Le secteur tertiaire en question, Paris, Economica, p. 124-128.

Picot, W.G., (1986), Canada's Industries :-Growth in Jobs Over Three Decades. A Review of the Changing Industrial Mix of Employment 1951-1986, Ottawa, Statistics Canada, Social \& Economic Studies Division, Ministry of Supplies and Services Canada.

Planque, B., (1982), Le développement décentralisé, Paris, Litec, Col. Gual. no 16.

Pottier, C., (1984), L'attitude des tissus industriels régionaux à promouvoir la révolution technologique, Milan Communication au 24ème Congrès Européen de la Regional Science Association.

Promotions Nordcom (les), (1987), Répertoire des gens d'affaires francophones, 2e Edition, 1987-1988, Sudbury, Les Promotions Nordcom Inc.

Rabier, J.C. et Sipek, K., (1985), «Résumé des discussions», dans J. de Bandt, (éd.), Les services dans les sociétés industrielles, Paris, Economica, p. 185-200.

Schon, et al., (1971), Beyond the Stable State, New York, Basic Books.

Siniscalco, D., (1985), «Emplois dans le secteur des services et changement structurel». dans J. de Bandt (éd.), Les services dans les sociétés industrielles, Paris Economica, p. 83-105.

Statistique Canada, «Distribution de l'emploi», Recensements de 1951 à 1986, Ottawa, Statistique Canada.

Tenehaus, M. et Young, F.W., (1985), «An analysis and synthesis of multiple correspondence analysis, optimal scaling, dual scaling, homogeneity analysis and other methods for quantifying categorical multivariate datas, Psychometrica, vol. 50, p. 429-47. 


\section{ANNEXE 1}

\section{Questionnaire partiel}

1. Quel est votre secteur d'activités ?
a) gouvernement
b) services
c) secteur manufacturier

2. Quelle est votre occupation ?
a) directeur
b) propriétaire
c) gérant/superviseur
d) professionnel/agent
e) autre

3. Combien d'employés votre compagnie occupe-t-elle en ce moment ?
tp) 1 à 5 - très petite
p) 6 à 15 - petite
m) 16 à 50 - moyenne
g) 51 à 199 - grande
tg) 200 et plus - très grande

4. Depuis combien de temps votre compagnie offre-t-elle ses services ?
tj) 1 à 5 ans - très jeune
j) 6 à 10 ans - jeune
m) 11 à 25 ans - moyenne
v) 26 ans et plus - vieille

5. De quel type est votre compagnie ?
a) société par actions
b) franchise
c) PME
d) gouvernement
e) coopérative
f) autre

6. Croyez-vous que le Nord-Ontarien a pris de l'importance quant à sa position dans le monde des affaires?
a) beaucoup
b) assez
c) très peu
d) pas du tout

7. Dans quelle mesure voyez-vous une croissance économique dans votre secteur?
a) beaucoup
b) assez
c) très peu
d) pas du tout

8. Jusqu'à quel point le Nord-Ontarien est-il favorable aux gens d'affaires francophones?
a) beaucoup
b) assez
c) très peu
d) pas du tout

13. En général, comment voyez-vous le développement économique du nord ?
a) excellent
b) stable
c) en déclin
d) indécis(e)

14. Quelles mesures devrions-nous adopter pour maintenir et/ou améliorer le développement de l'économie nord-ontarienne?

15. Quels secteurs seront, d'après vous, les futurs générateurs d'emplois dans l'économie nord-ontarienne?
a) forestier
b) minier
c) touristique
d) gouvernemental
e) vente au détail
f) finance
g) restauration
h) secteur manufacturier
i) transports
j) autres (spécifiez). 